\title{
Quantitative Ultrasound Differentiates Brain and Brain Tumour Phantoms
}

\author{
Hannah Thomson \\ Centre for Medical and Industrial \\ Ultrasonics \\ University of Glasgow \\ Glasgow, Scotland \\ h.thomson.3@research.gla.ac.uk \\ Mitch Baldwin \\ Stryker Medical Intruments \\ Global Headquarters \\ Kalamazoo, USA \\ Mitch.Baldwin@stryker.com
}

\author{
Shufan Yang \\ Centre for Medical and Industrial \\ Ultrasonics \\ University of Glasgow \\ Glasgow, Scotland \\ Shufan.Yang@glasgow.ac.uk
}

\author{
Helen Mulvana \\ Biomedical Engineering \\ University of Strathclyde \\ Glasgow, Scotland \\ helen.mulvana@strath.ac.uk
}

\author{
Thomas Stritch \\ Stryker Medical Intruments \\ Innovation Centre \\ Cork, Ireland \\ Thomas.Stritch@stryker.com
}

\author{
Sandy Cochran \\ Centre for Medical and Industrial \\ Ultrasonics \\ University of Glasgow \\ Glasgow, Scotland \\ Sandy.Cochran@glasgow.ac.uk
}

\begin{abstract}
The acoustic impedance and attenuation of chicken liver and gizzard muscle were measured over a frequency range of $1-10 \mathrm{MHz}$. The results validated their use as ultrasound phantoms for brain and brain tumour respectively. These tissues were then used as an initial test of whether quantitative ultrasound (QUS) could differentiate between brain and brain tumour tissue-like materials. QUS is a technique which infers information about tissue microstructure, such as effective scatterer size (ESD) and acoustic concentration, through the backscattered power spectrum of insonated tissues. The ESD of the brain tumour phantom was significantly higher than that of the healthy brain phantom, $(87.3 \pm 8.6 \mu \mathrm{m}$ vs $61.2 \pm 5.8$ $\mu \mathrm{m})$. The distinction in scattering properties shows potential to use QUS in soft tissue cancer detection.

Keywords - Quantitative Ultrasound, Tissue Phantoms, Tissue Characterisation, Finite Element Analysis
\end{abstract}

\section{INTRODUCTION}

Conventional intraoperative ultrasound (iUS) has proven to be a useful adjunct in neuro-oncology, where realtime images can guide the surgeon to the correct location after craniotomy [1]. However, in glioma surgery, image quality decreases dramatically due to the limited acoustic contrast between brain and both low and high grade glioma [2]. Quantitative ultrasound (QUS) infers properties about tissue microstructure from the backscattered radiofrequency (RF) data and has been shown to enhance the diagnostic capabilities of conventional ultrasound [3]. Small inhomogeneities, typically with dimensions less than the wavelength, $\lambda$, in tissues cause ultrasound waves to scatter, with the extent of scattering dependent on the size of the acoustic inhomogeneities. It is hypothesised than cancerous brain tissue will scatter ultrasound differently to healthy brain tissue due to underlying microstructure e.g. number and spatial correlation of cells in each tissue.

Scattering from single spheres and cylinders was first described mathematically by Faran in 1953 [4]. Isana et al. extended this to show that tissue modelled with acoustic impedance $\mathrm{Z}$ with small fluctuations from its mean value, $\mathrm{Z}_{0}$, following a Gaussian spatial auto-correlation function will generate a power spectrum which is dependent on the fourth power of the frequency of the ultrasound source

Techniques measuring the backscattered ultrasound have shown potential in characterising different aspects of

This work was supported by the UK Engineering and Physical Sciences

Research Council (EPSRC) and Stryker Medical Devices under the project number.

XXX-X-XXXX-XXXX-X/XX/\$XX.00 @20XX IEEE microstructure of tissues [5][6]. Early studies included work from Felleppa et al. who used the frequency-dependent backscatter to obtain estimates of scatterer size and concentration from ocular tumour [7]. Mamou et al. successfully characterised cancerous lymph nodes using this technique and found that metastatic nodes had an ESD larger than that in cancer-free nodes [8]. Similar results can be seen through various other studies, notably by Oelze et al., who illustrated that overall estimates of scatterer size were $44 \%$ larger inside rat mammary tumours than outside in an in vivo study [9]. Recently, it was shown that techniques extracting spectral parameters from healthy and cancerous tissues and implementing a machine learning algorithm showed a high sensitivity and specificity for identifying for various types of cancerous tissue, further highlighting the diagnostic potential [10].

In the present study, two acoustic phantoms were used to test the feasibility of quantitative ultrasound to differentiate between brain and brain tumour intraoperatively. Acoustic phantoms are materials which exhibit similar acoustic and mechanical properties to human tissue. They are widely used both for training sonographers and in quality assurance monitoring for new ultrasound systems. For a phantom to be valid, it should have similar acoustic properties to the tissue it is designed to mimic, over the frequency range of interest. These include mechanical properties, acoustic velocity, attenuation coefficient and acoustic impedance.

\section{METHODS}

\section{A. Acoustic Impedance}

Fresh chicken liver and gizzard muscle were purchased from a local Halal butcher. This tissue was chosen as Stewart et al. found them to have similar mechanical properties to brain and brain tumour [11]; however, they did not measure the acoustic characteristics. The samples were cut into small slices of varying sizes with a scalpel, taking care that the thickness of the slices was as uniform as possible (Fig. 1a). The density of the tissue was calculated by measuring the mass, then estimating the volume via the Archimedes Principle. To calculate the speed of sound, the tissue was placed on a single element $10 \mathrm{MHz}$ transducer (unfocused immersion transducer, Olympus, aperture $10 \mathrm{~mm}$ ). Each sample was placed on a sample holder, which was designed to surround the transducer and allow the sample to sit parallel to the transducer face. 
The transducer was connected to an ultrasonic pulserreceiver (DPR300, JSR - Imaginant, NY, USA) operating in pulse-echo mode and the receiver output was connected to an oscilloscope. Once a steady reflection pattern was seen, the time, $\Delta \mathrm{t}$, between successive echoes was measured using the oscilloscope measuring function. The speed of sound, $\mathrm{c}$, in the tissue can then be calculated by:

$$
c=\frac{2 d}{\Delta t}
$$

where $\mathrm{d}$ is the thickness of the sample. This procedure was repeated for twelve samples of both liver and gizzard and the average values were taken.

\section{B. Acoustic Attenuation}

The attenuation coefficient is the acoustic energy lost via scattering, absorption and other mechanisms when an ultrasound pulse propagates through a medium [12]. The method used to calculate the attenuation was based on the transmission loss method published by the National Physical Laboratory [13]. In summary, output readings are recorded before and after a tissue sample is placed in the acoustic path between two transducers, to calculate the energy lost.
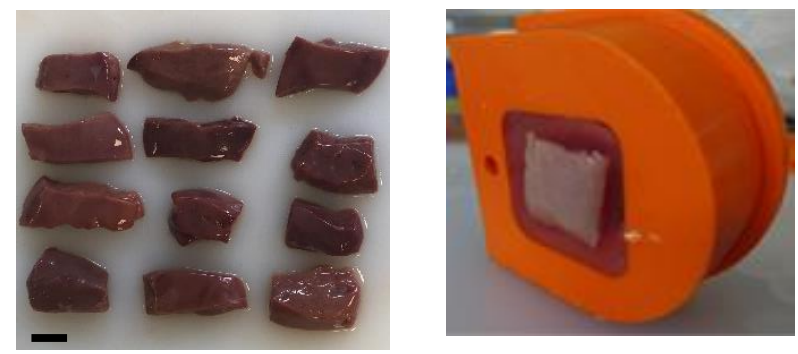

Figure 1: Acoustic characterisation measurements (a) Samples of chicken liver. (b) Sample placed into custom made $3 D$ printed holder, encased in agar.

A needle hydrophone $(0.5 \mathrm{~mm}$ diameter, with $8 \mathrm{~dB}$ preamplifier, Precision Acoustics Ltd, UK) was positioned at the focal point of the transducer in a tank of degassed water. The empty sample holder was placed in the water tank and measurements were taken for the reference voltage, $\mathrm{V}_{\mathrm{W}}$. Next, a tissue sample enclosed in agar was placed in the sample holder (Fig. 1b) and a reduced voltage reading was recorded on the hydrophone, $\mathrm{V}_{\text {SAMPLE. }}$ This procedure was carried out three times for each sample.

The estimated thickness of the tissue was calculated by completing a pulse echo-test on the sample with the speed of sound now known from the previous experiment. The attenuation coefficient for the specific frequency could then be calculated by:

$$
a_{T I S}=\frac{1}{d}\left[20 \log _{10} \frac{V_{W}}{V_{S A M P L E}}-\tilde{a}_{A G A R}\right]+a_{W}
$$

where:

$$
\begin{aligned}
& a_{\text {TIS }}=\text { Attenuation in tissue }(\mathrm{dB} / \mathrm{cm}) \\
& \mathrm{d}=\text { Thickness of tissue }(\mathrm{cm}) \\
& \mathrm{V}_{\mathrm{W}}=\text { Signal through water path without tissue }(\mathrm{V}) \\
& \mathrm{V}_{\mathrm{SAMPLE}}=\text { Signal with tissue and agar present }(\mathrm{V}) \\
& \tilde{\mathrm{a}}_{\text {AGAR }}=\text { Attenuation due to agar }(\mathrm{dB})
\end{aligned}
$$

\section{QUS Parameters}

Six tissue samples of uniform thickness were prepared and placed in degassed water at the natural focal distance of a transducer (Fig. 2). Again, a $10 \mathrm{MHz}$ transducer connected to the JSR pulser-receiver was used for this study. A reference measurement was also made, with the tissue replaced with a quartz plate, as this is required for calibration.

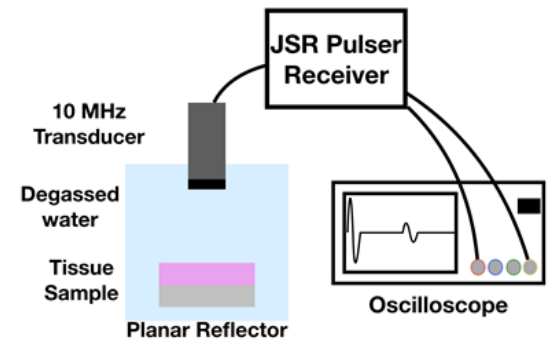

Figure 2: Experimental set-up to obtain backscattered power spectrum from tissue

The backscattered power spectrum is estimated experimentally from the following formula [9]:

$$
W_{\text {meas }}(f)=\frac{1}{N} \frac{\Re^{2}}{4} \sum_{n=1}^{N} \frac{\left|F T\left\{\rho_{n}(t)\right\}\right|^{2}}{W_{\text {ref }}(f)}
$$

where:

$$
\begin{aligned}
& \Re=\text { reflection coefficient of the quartz flat } \\
& \mathbf{N}=\text { number of scans } \\
& \rho_{n}=\text { RF data from scattering from the nth A-scan, } \\
& \text { gated by a Hanning window. } \\
& W_{\text {ref }}=\text { power spectrum reflected from a quartz flat }
\end{aligned}
$$

A gate length of $7 \mathrm{~mm}$ was used in this study and the tissue samples had a thickness of $1 \mathrm{~cm}$ on average. Eight scans were taken per sample. In order to obtain an accurate power spectrum resulting from only scattering effects, attenuation must be taken into account by multiplying by a compensation term [14]:

$$
W_{\text {comp }}(f)=W_{\text {meas }}(f) e^{4 \alpha_{0}(f) x_{0}}\left[\frac{2 \alpha(f) L}{1-e^{-2 \alpha(f) L}}\right]^{2}
$$

where:

$$
\begin{aligned}
& \alpha_{0}=\text { attenuation coefficient of water }(\mathrm{dB} / \mathrm{cm}) \\
& x_{0}=\text { propagation distance in water }(\mathrm{cm}) \\
& \alpha=\text { attenuation coefficient in the gated region } \\
& \text { (assumed constant } \mathrm{dB} / \mathrm{cm})
\end{aligned}
$$

The measured compensated power spectrum was compared to the power spectrum expected from extending Faran's theory of scattering to a distribution of scatters [15], [16]

$$
W(f)=\frac{185 L q^{2} a^{6} \rho z_{v a r}^{2} f^{4}}{\left[1+2.66(f q a)^{2}\right]} e^{-12.159 f^{2} a^{2}}
$$

where:

$$
\begin{aligned}
& a_{\text {eff }}=\text { Effective Scatter Diameter }(\mathrm{mm}) \\
& \mathrm{L}=\text { gate length }(\mathrm{mm}) \text { (the size of ROI) } \\
& \mathrm{q}=\text { ratio of transducer aperture radius to distance to } \\
& \text { ROI } \\
& \mathrm{f}=\text { frequency }(\mathrm{MHz}) \\
& \rho z_{\text {var }}^{2}=\text { 'acoustic concentration' i.e. a product of the }
\end{aligned}
$$


number of scatterers per $\mathrm{mm}^{3}$ and $z_{v a r}=\left(\mathrm{Z}-\mathrm{Z}_{0}\right) / \mathrm{Z}_{0}$, a measure of the relative acoustic impedance of the scatterers $(Z)$ and the surrounding medium $\left(Z_{0}\right)$

The parameters of interest $\left(a_{e f f}, \rho z_{v a r}^{2}\right)$ are found by a technique first described in detail by Oelze et al. to minimise the average squared difference between the theoretical normalised power spectrum and $W_{\text {comp }}(f)$ [9]. This involves several expansions and assumptions (such as q < 1) which are fulfilled in this experimental set-up. Ultimately, a straight line is estimated using the terms on the left hand side of Eq. 6.

$$
\begin{aligned}
& 10 \log \left(W_{\text {comp }}(f)\right)-10 \log f^{4} \approx \\
& M\left(a_{\text {eff }}^{2}\right) f^{2}+I\left(a_{\text {eff }}^{6}, \rho z_{\text {var }}^{2}\right)
\end{aligned}
$$

Once the gradient and intercept of the straight line are estimated, the ESD, $a_{e f f}^{2}$, and acoustic concentration, $\rho z_{v a r}^{2}$, can be calculated from:

$$
\begin{gathered}
M=-4.34\left[12.159+2.66 q^{2}\right] a_{e f f}^{2} \\
I=10 \log \left[185 L q^{2} a_{e f f}^{2} \rho z_{v a r}^{2}\right]
\end{gathered}
$$

Finite element analysis simulations were carried out using PZFlex (Oscale, CA, USA) to test the accuracy of the estimation scheme. The set up for the $2 \mathrm{D}$ in silico model was made to emulate a pulse-echo test, as in the experimental measurements. It simulates a $10 \mathrm{MHz}$ single cycle sinusoid travelling through $2 \mathrm{~mm}$ of water then $2 \mathrm{~mm}$ of tissue with embedded scatterers (Fig. 3a). The acoustic impedance of the scatterers was $10 \%$ higher than the surrounding medium.
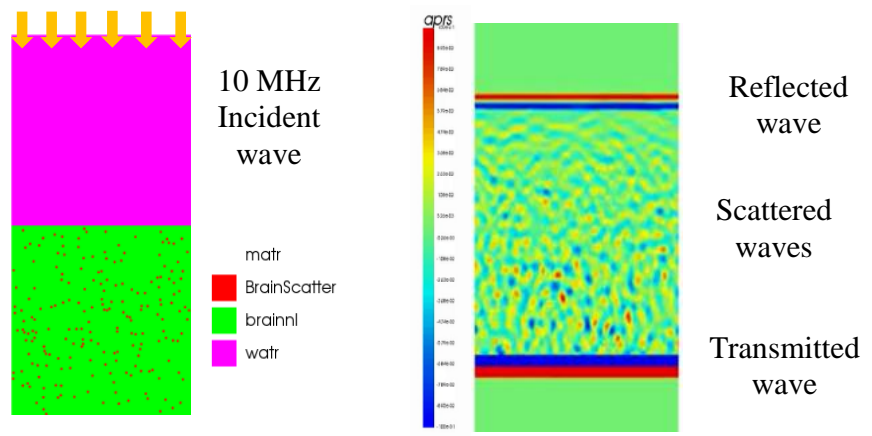

Figure 3 (a) Typical model, scatterer size $50 \mu$ m and 50 scatterers/ $\mathrm{mm}^{2}$ (b) Screenshot of simulation showing acoustic pressure wave interaction with scatterers

The scatterer size was increased from $10-100 \mu \mathrm{m}$ in $10 \mu \mathrm{m}$ intervals. Ten models were created for each scatterer size to study the effect of random spatial distributions.

\section{RESULTS}

\section{A. Acoustic Impedance}

The average results for the density, acoustic velocity and acoustic impedance are shown in Table 1.

When compared with results in the literature [17], most tumours and healthy brain tissue fall in the density range $1040-1050 \mathrm{~kg} \mathrm{~m}^{-3}$. Chicken liver was found to have a slightly higher density than one would expect for healthy brain tissue; however, the results for chicken gizzard appear accurate. The velocity values for both tissues agree well with the literature for healthy brain and glioblastoma $[17,18]$.

TABLE I. ACOUSTIC IMPEDANCE OF LIVER AND GIZZARD

\begin{tabular}{|l|c|c|c|}
\hline \multicolumn{1}{|c|}{ Tissue } & $\begin{array}{c}\text { Density } \\
\left(\boldsymbol{k g ~ m}^{-3}\right)\end{array}$ & $\begin{array}{c}\text { Acoustic } \\
\text { velocity }\left(\boldsymbol{m ~ s}^{-1}\right)\end{array}$ & $\begin{array}{c}\text { Acoustic } \\
\text { Impedance } \\
\text { (MRayl) }\end{array}$ \\
\hline $\begin{array}{l}\text { Chicken } \\
\text { Liver }\end{array}$ & $1067 \pm 23$ & $1539 \pm 85$ & $1.64 \pm 0.06$ \\
\hline $\begin{array}{l}\text { Chicken } \\
\text { Gizzard }\end{array}$ & $1051 \pm 8$ & $1510 \pm 44$ & $1.59 \pm 0.03$ \\
\hline
\end{tabular}

\section{B. Acoustic Attenuation}

The attenuation for both tissues over the range $1-10 \mathrm{MHz}$ are displayed in Fig. 4.

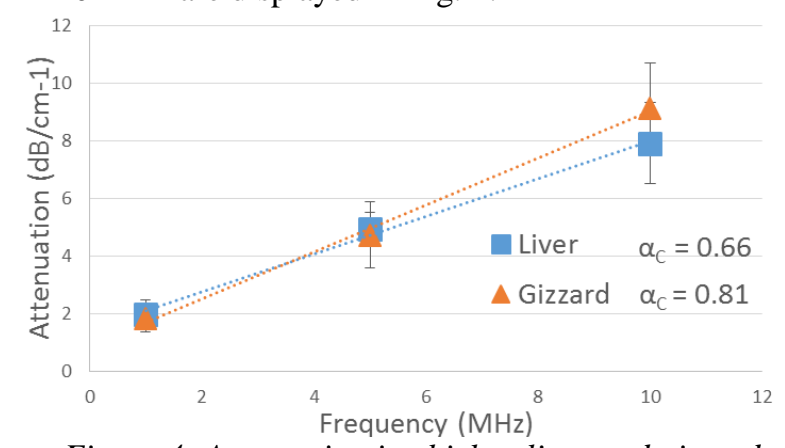

Figure 4: Attenuation in chicken liver and gizzard

The attenuation coefficient (measured in $\mathrm{dB} \mathrm{cm}^{-1} \mathrm{MHz}^{-1}$ ) was found to be $0.66 \pm 0.16$ and $0.81 \pm 0.18$ for chicken liver and gizzard respectively. When compared to healthy brain in vivo, the results for liver agree well at $5 \mathrm{MHz}$ and we would expect to see further agreement if the data were extrapolated to $10 \mathrm{MHz}$ [19]. Chicken gizzard agrees well with the ex vivo results for glioblastoma, further evidencing its suitability as a malignant tumour phantom [18]. The results emphasise the similarity between the two tissue types in terms of acoustic concentration and the error analysis highlights the heterogeneity of the tissue samples. It should be noted that the main source of error in these analyses arose from the accuracy with which tissue samples could be prepared to achieve the desired thickness.

\section{QUS Parameters}

Initial estimates of the ESD and acoustic concentration $\left(a_{e f f}, \rho z_{v a r}^{2}\right)$ show a distinct difference between the brain and brain tumour phantoms. The average scatterer sizes were found to be $87.3 \pm 8.6 \mu \mathrm{m}$ vs $61.2 \pm 5.8 \mu \mathrm{m}$ in chicken gizzard and liver respectively (Fig. 5).

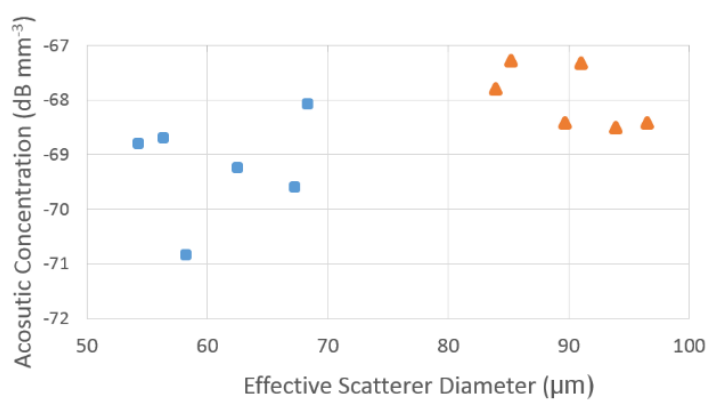

Figure 5: Spectral slope and intercept for liver (healthy brain phantom, blue squares) and gizzard (cancerous brain phantom, red triangles) 
The increase in scatterer size in cancerous tissue phantom is an interesting result; the same trend was reported in the literature when this technique was used in ex-vivo healthy and cancerous tissue samples [10]. The acoustic concentration was found to be similar in both tissues, implying a similar number of scatterers per unit volume in each tissue. The variance of the results could be minimised by taking a larger number of scans to contribute to the average power spectrum.

The accuracy of the scatterer diameter estimation was tested using an in silico study. The ESD was found for each of the 10 geometries using the technique described above, corresponding to scatterer sizes varying from $10-100 \mu \mathrm{m}$. It can be seen from the results (Fig. 9) that this technique provided good accuracy at estimating the ESD in the simulation when ESD $>40 \mu \mathrm{m}$. This gives confidence in the reliability of the results derived from experimental data. However, it can also be seen that it is hard to distinguish scatterers with ESD $10-30 \mu \mathrm{m}$, which may be due to the effects of the mesh size of the model.

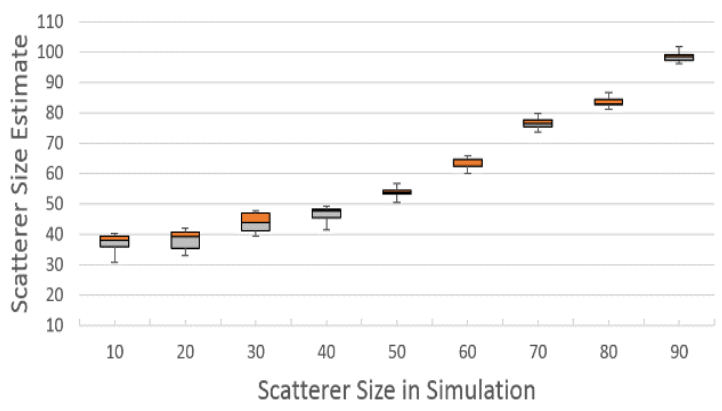

Figure 6: Spectral gradient vs diameter, average of 10 simulations. Error bars show standard deviation.

\section{DISCUSSION AND CONCLUSION}

The acoustic properties of chicken liver and gizzard have been evaluated and the results correspond with those reported in the literature for acoustic velocity and attenuation for healthy brain tissue and malignant brain tumour respectively. However, it is known that the acoustic properties of brain tumours differ depending on malignancy, so this phantom would not be valid for all tumour types. It is also important to note that a good macroscopic acoustic phantom does not necessarily indicate a good microscopic acoustic phantom. Initial results indicate that QUS shows promise as a tool for differentiating the two tissue types of interest here. These tissues are hard to differentiate using conventional ultrasound; however their microstructure is so different that it causes ultrasound to scatter very differently within the tissue. This study indicates that spectrum based parameters are essential for differentiation of tissues indistinguishable by conventional ultrasound. Display of parametric images simultaneously with B-mode images will be necessary for intraoperative use; we plan to use spectral parameters from a large number of ex vivo tissue samples and in silico models to train a binary classifier to demonstrate this approach.

\section{ACKNOWLEDGMENTS}

The authors are pleased to acknowledge Dani Assi (MSc student) and Hannah Gibson (summer student) who worked with the author in the Centre for Medical and Industrial Ultrasonics, University of Glasgow, to solve some problems encountered in this project.

\section{REFERENCES}

[1] J. Coburger et al., "Linear array ultrasound in low-grade glioma surgery: histology-based assessment of accuracy in comparison to conventional intraoperative ultrasound and intraoperative MRI," Acta Neurochir. (Wien)., vol. 157, no. 2, pp. 195-206, 2015.

[2] R. Sastry et al., "Applications of Ultrasound in the Resection of Brain Tumors," J. Neuroimaging, vol. 27, no. 1, pp. 5-15, 2017.

[3] D. P. Hruska, J. Sanchez, and M. L. Oelze, "Improved diagnostics through quantitative ultrasound imaging," Proc. 31st Annu. Int. Conf. IEEE Eng. Med. Biol. Soc. Eng. Futur. Biomed. EMBC 2009, pp. 1956-1959, 2009.

[4] J. J. Faran, "Sound Scattering by Solid Cylinders and Spheres," vol. 405, no. 1951,1988

[5] F. L. Lizzi, M. Ostromogilsky, E. J. Feleppa, M. C. Rorke, and M. M. Yaremko, "Relationship of Ultrasonic Spectral Parameters to Features of Tissue Microstructure," no. 3, 1987.

[6] M. F. Insana and T. J. Hall, "Parametric Ultrasound Imaging from Backscatter Coefficient Measurements: Image Formation and Interpretation," Ultrason. Imaging, vol. 12, no. 4, pp. 245-267, 1990. [7] M. L. Oelze, W. D. O'Brien, J. P. Blue, and J. F. Zachary, "Differentiation and characterization of rat mammary fibroadenomas and 4T1 mouse carcinomas using quantitative ultrasound imaging," IEEE Trans. Med. Imaging, vol. 23, no. 6, pp. 764-771, 2004.

[8] J. Mamou and M. L. Oelze, Quantitative Ultrasound in Soft Tissues, 1st ed. Springer, 2013.

[9] E. J. Feleppa, F. L. Lizzi, D. J. Coleman, and M. M. Yaremko, "Diagnostic spectrum analysis in ophthalmology: A physical perspective," Ultrasound Med. Biol., vol. 12, no. 8, pp. 623-631, 1986.

[10] J. Mamou et al., "Three-Dimensional High-Frequency Characterization of Cancerous Lymph Nodes," Ultrasound Med. Biol., vol. 36, no. 3, pp. 361-375, 2010.

[11] M. L. Oelze, J. F. Zachary, and W. D. O'Brien, "Characterization of tissue microstructure using ultrasonic backscatter: Theory and technique for optimization using a Gaussian form factor," J. Acoust. Soc. Am., vol. 112 , no. 3, pp. 1202-1211, 2002.

[12] D. Rohrbach, B. Wodlinger, J. Wen, J. Mamou, and E. Feleppa, "HighFrequency Quantitative Ultrasound for Imaging Prostate Cancer Using a Novel Micro-Ultrasound Scanner," Ultrasound Med. Biol., vol. 44, no. 7, pp. 1341-1354, 2018.

[13] D. C. Stewart, A. Rubiano, K. Dyson, and C. S. Simmons, "Mechanical characterization of human brain tumors from patients and comparison to potential surgical phantoms," PLoS One, vol. 12, no. 6, pp. 1-19, 2017.

[14] P. Hoskins, Diagnostic Ultrasound: Physics and Equipment. Greenwich Medical Media, 2003.

[15] S. Rajagopal, N. Sadhoo, and B. Zeqiri, "Reference Characterisation of Sound Speed and Attenuation of the IEC Agar-Based Tissue-Mimicking Material Up to a Frequency of 60MHz," Ultrasound Med. Biol., vol. 41, no. 1, pp. 317-333, 2015.

[16] A. Manuscript, "Attenuation Slope for Quantitative Ultrasound Imaging," vol. 35, no. 2, pp. 162-172, 2013.

[17]F. A. Duck, Physical Properties of Tissues: A Comprehensive Reference Book. Academic Press, 2013.

[18] W. Schiefer, E. Kazner, and K. Zülch, "Proceedings in EchoEncephalography: International Symposium on Echo-Encephalography," 1968.

[19] M. A. S. Trowitzki, S. E. B. Rand, and K. L. I. J. Enderka, “Ultrasonic Radio-Frequency Spectrum Analysis of Normal Brain Tissue," vol. 33, no. 4, pp. 522-529, 2007. 
